\section{Imprinting: the Achilles heel of trio-based exome sequencing}

The possibilities and application of molecular genetics diagnostics have increased immensely in the past decade, particularly in the field of rare syndromes causing intellectual disability (ID). In many cases the most important reason for parents to have genetic diagnostics performed is to determine their recurrence risk (RR) for a future pregnancy and the RR for other family members. Fortunately, the vast majority of ID has been shown to be caused by de novo mutations, ${ }^{1-3}$ which result in a low RR. A minority of ID is caused by mutations in recessive or X-linked genes, with an associated 25\% RR. Mutations and chromosomal deletions in imprinted regions, although rare, are a well-known cause of ID (e.g., on chromosome 15, causing Angelman and Prader-Willi syndromes) and may result in a $50 \%$ RR for parents and siblings of the carrier parent. However, familial mutations in imprinted genes leading to severe ID have only rarely been reported in the medical literature, probably because most of those mutations also arise de novo.

Next-generation sequencing is rapidly replacing classic molecular diagnostics. It has been shown that trio-based sequencing (sequencing both the patient and parents) is an efficient way of finding causal mutations. Importantly, whereas all exome sequencing studies published to date report a focus on de novo, X-linked, and recessive inheritance, none report taking familial imprinted mutations explicitly into account in their filtering strategy, ${ }^{1-3}$ and none report finding an imprinted cause of ID. We found in the literature only a single report of an imprinted mutation being identified in a diagnostic setting. ${ }^{4}$

We encountered a large family with 14 affected individuals from five nuclear families in two generations. ${ }^{5}$ All patients had severe ID, and many of them displayed camptodactyly and hypotonia. There were dysmorphic features, but no syndromic diagnosis could be made by experienced clinical geneticists. Four of the patients died at a young age as a result of various complications. Exome sequencing was accidentally performed in two centers: in one center a trio design was chosen; in the other center, a single patient was sequenced. No pathogenic mutation was identified by either center. Later analysis in a research setting - taking into account the extraordinary pedigree of this family, showing that all affected individuals were linked though their fathers-identified a paternally inherited pathogenic frameshift mutation (NM_019066.4:c.1996dup (p.(Gln666Profs $\left.\left.{ }^{\star} 47\right)\right)$ in MAGEL2. This variant was missed in the trio exome because it was inherited by the father (and thus did not adhere to de novo, recessive, or X-linked inheritance), and it was ignored in the single exome because the gene was not on the ID gene list and focus was given to recessive inheritance because this was considered more likely.

MAGEL2 is located in the imprinted Prader-Willi/Angelman region on chromosome 15. De novo truncating mutations in MAGEL2 on the paternal allele was shown to cause a PraderWilli-like phenotype in four patients. ${ }^{6}$ The diagnosis of PraderWilli syndrome was considered in one of our patients, although it was not in the differential diagnosis for the other patients.

It is worrisome to realize that the causative mutation was identified only because of the extended pedigree of this family. Missing the diagnosis would have led to more affected individuals in the family, given the associated 50\% RR. Indeed, for this family in particular, the consequences of this mutation have been devastating and will most likely not end here because several other nuclear families are at risk. To further investigate the scope of this potential pitfall, we looked for putative loss-of-function mutations in MAGEL2, UBE3A, and KCNK9 in the Exome Aggregation Consortium database (http:// exac.broadinstitute.org; accessed 23 June 2016) consisting of $\sim 120,000$ alleles. We identified six confident loss-of-function alleles (one in MAGEL2, four in UBE3A, and one in KCNK9), leading to an estimated carrier frequency of $\sim 1$ in 10,000.

This case shows that this issue arises not only when trio exome sequencing is performed but perhaps also when only the affected individual is sequenced, which has been shown to be another effective strategy. ${ }^{7}$ In-depth analysis of the failure to identify a mutation in a single exome reveals that it occurs mostly because too many variants remained, leading to difficulties in assessing the merit of each variant. We therefore recommend that any sequencing analysis pipeline take imprinted genes explicitly into account, displaying all variants-inherited or not-in this relatively limited number of genes.

\section{ACKNOWLEDGMENTS}

The authors acknowledge helpful discussions with Sander Stegmann, Rolph Pfundt, Han Brunner, Sarina Kant, Els Peeters, Connie Stumpel, and Gert-Jan van Ommen.

\section{DISCLOSURE}

The authors declare no conflict of interest.

Emmelien Aten, $M D, P h D^{1}$, Michael D. Fountain, $P h D^{2,3}$, Arie van Haeringen, $M D^{1}$, Christian P. Schaaf, $M D, P h D^{2,3}$ and Gijs W.E. Santen, $M D, P h D^{1}$

${ }^{1}$ Department of Clinical Genetics, Leiden University Medical Center, Leiden, The Netherlands; ${ }^{2}$ Department of Molecular and Human Genetics, Baylor College of Medicine, Houston, Texas, USA; ${ }^{3}$ Jan and Dan Duncan Neurological Research Institute, Texas Children's Hospital, Houston, Texas, USA. Correspondence: Gijs W.E. Santen (Santen@lumc.nl) 


\section{LETTER TO THE EDITOR}

\section{REFERENCES}

1. de Ligt J, Willemsen $\mathrm{MH}$, van Bon BW, et al. Diagnostic exome sequencing in persons with severe intellectual disability. N Engl J Med 2012;367:1921-1929.

2. Rauch A, Wieczorek D, Graf E, et al. Range of genetic mutations associated with severe non-syndromic sporadic intellectual disability: an exome sequencing study. Lancet 2012;380:1674-1682.

3. Wright CF, Fitzgerald TW, Jones WD, et al.; DDD study. Genetic diagnosis of developmental disorders in the DDD study: a scalable analysis of genome-wide research data. Lancet 2015;385:1305-1314.

4. Bodian DL, Solomon BD, Khromykh A, et al. Diagnosis of an imprinted-gene syndrome by a novel bioinformatics analysis of whole-genome sequences from a family trio. Mol Genet Genomic Med 2014;2:530-538.
5. Fountain MD, Aten E, Cho MT, et al. The phenotypic spectrum of Schaaf-Yang syndrome: 18 new affected individuals from 14 families. Genet Med; e-pub ahead of print 16 May 2016.

6. Schaaf CP, Gonzalez-Garay ML, Xia F, et al. Truncating mutations of MAGEL2 cause Prader-Willi phenotypes and autism. Nat Genet 2013;45:1405-1408.

7. Yang Y, Muzny DM, Reid JG, et al. Clinical whole-exome sequencing for the diagnosis of mendelian disorders. N Engl J Med 2013;369:1502-1511.

Advance online publication 15 September 2016. doi:10.1038/gim.2016.128 